\title{
Wickerhamomyces anomalus
}

National Cancer Institute

\section{Source}

National Cancer Institute. Wickerhamomyces anomalus. NCI Thesaurus. Code C114262.

A yeast in the phylum Ascomycota. This species is spheroidal to elong ate shaped, forms pseudohyphae and produces hat-shaped ascospores, can grow anaerobically, ferments glucose and sucrose, assimilates glucose, galactose, fructose, raffinose and mannose but not formic acid and methanol, and utilizes nitrate and urea. W. anomalus is used in food and feed processing and functions as a biopreservation agent, phytase producer, and for folate biofortification. It is also the causative agent of opportunistic mycoses that develop in immunocompromised individuals as well as neonates. 